\section{Carcinoma de Tireóide Pouco Diferenciado: Novas Considerações Terapêuticas}

\section{RESUMO}

Para a maioria dos carcinomas diferenciados de tireóide, como o papilífero e o folicular, após a tireoidectomia total e ${ }^{131}$ | para ablação de remanescentes tireoideanos, o tratamento com hormônios tireoideanos para suprimir os níveis de TSH reduz o crescimento de qualquer célula cancerosa remanescente, e o tratamento com radiação específica para as células cancerosas cura ou controla muito bem a doença. Os carcinomas de tireóide são considerados pouco diferenciados quando começam a perder as funções de captação de iodo e a dependência do TSH para crescimento e produção de proteínas tireóide-específicas, como proteína NIS, tireoglobulina e desiodases. Um dos maiores desafios no manejo de pacientes com carcinoma de tireóide derivados das células foliculares é o tratamento de tumores que evoluem apesar da cirurgia, ${ }^{131}$ I e supressão do TSH com T4. Com o melhor conhecimento da sinalização molecular anormal nas células tireoideanas cancerosas, atualmente novas terapias dirigidas a alvos moleculares específicos envolvidos na transformação neoplásica têm sido utilizadas. Com a identificação das necessidades moleculares críticas para a iniciação, manutenção e progressão tumoral, terapias combinadas com agentes terapêuticos alvo-dirigidos, agindo em cada uma destas etapas, irão melhorar o tratamento do carcinoma pouco diferenciado de tireóide. (Arq Bras Endocrinol Metab 2005;49/5:711718)

Descritores: Carcinoma pouco diferenciado; Sinalização molecular; Drogas alvo-dirigidas

\section{ABSTRACT}

Poorly Differentiated Thyroid Carcinomas: New Therapeutics Considerations.

For most differentiated thyroid carcinomas, as papillary and follicular carcinomas, following total thyroidectomy and 131 I therapy for thyroid remnant ablation, treatment with thyroid hormones to suppress TSH levels will reduce the growth of any remaining thyroid cancer cells, and thyroid cell-specific radiation therapy will either cure or control the disease. Thyroid carcinomas are considered poorly differentiated when they start to lose such functions as iodine uptake and thyrotropin-dependence for growth and production of thyroid proteins like NIS, thyroglobulin and desiodases. One of the greatest challenges in the management of patients with follicular cell-derived thyroid cancer is the treatment of tumors that progressed despite surgery, 1311 and T4 suppression of TSH. With the better knowledge of the abnormal molecular signaling in thyroid cancer cells, actually known targeted cancer therapies, directed against molecules involved in neoplastic transformation, are being used. As the critical molecular requirements for tumor initiation, maintenance and progression are identified, combination therapies with targeted agents acting on each of them will improve the treatment of poorly differentiated thyroid carcinoma. (Arq Bras Endocrinol Metab 2005;49/5:711-718)

\section{atualização}

\author{
Hans Graf
}

Serviço de Endocrinologia e Metabologia (SEMPR), Universidade Federal do Paraná, Curitiba, PR.

Recebido em 14/07/05

Revisado em 20/07/05 Aceito em 26/07/05 
Keywords: Poorly differentiated carcinoma; Molecular signaling; Targeted drugs

C CARCinoma diferenciado de tireóide, (CDT), papilífero (CPT) ou folicular (CFT), se caracteriza por apresentar uma série de funções que são encontradas na célula tireoideana normal. Por exemplo, a maioria dos CDT também expressam proteínas que são normalmente expressas na célula folicular, como a tireoglobulina, e a NIS (natrium sodi um symporter, "simportadora"). Em função disto, o paciente com carcinoma papilífero e folicular de tireóide, bem diferenciado, após o tratamento convencional inicial de tireoidectomia total, é normalmente submetido à ablação de remanescentes tireoideanos com ${ }^{131}$ I e com uma certa freqüência é submetido, no seguimento, a doses terapêuticas de 131I para tratamento de metástases. Em função disto, é possível obter remissão permanente na maioria dos pacientes e em quase $50 \%$ daqueles com metástases a distância (1-3). Esta ação actínica do 131I só é possível porque, no CDT, a expressão adequada da proteína NIS possibilita a entrada do 131I na célula folicular e a sua subseqüente ação destrutiva. Numa pequena porcentagem de pacientes, o carcinoma originado das células foliculares de tireóide não expressa ou perde a expressão adequada de proteínas. Neste caso, o prognóstico e evolução costumam ser piores em função da não captação de ${ }^{131}$ I pelas recidivas locais ou metástases a distância (4). Quando estas lesões não são capazes de retirada cirúrgica, outras estratégias terapêuticas precisam ser instituídas, como radioterapia externa, embolização arterial, quimioterapia isolada, combinações de drogas "inteligentes" e mesmo terapia gênica. Com o melhor conhecimento das vias moleculares de sinalização celular observamos o desenvolvimento de novas drogas dirigidas para etapas moleculares específicas envolvidas na progressão do câncer de tireóide (5). No momento existem várias drogas, de atuação celular bem diferente, sendo testadas no câncer de tireóide, tanto in vitro e in vivo, com trabalhos clínicos em fase I e II (5). A quimioterapia convencional se mostrou ineficaz para a maioria dos pacientes com metástases de tireóide em progressão. Entre várias drogas quimioterápicas, a resposta foi em média de 30\% em várias séries (6-11). Infelizmente, as respostas são parciais e de curta duração $(11,12)$. O endocrinologista, principal envolvido no tratamento do câncer de tireóide, deve conhecer as novas tendências da pesquisa oncológica e assumir um papel ativo nesta área. Nas duas últimas décadas, foram desvendados alguns dos mecanismos do desenvolvimento e progressão do câncer de tiróide, como a hiperexpressão ou ativação descontrolada do receptor tirosina-quinase, de moléculas sinalizadoras na cascata de sinalização e inibição da apoptose. Além disto, os fatores responsáveis pela progressão tumoral, angiogênese e disseminação metastática têm sido intensamente investigados (5).

\section{DROGAS QUE PROMOVEM A REDIFERENCI- AÇÃO}

Esta categoria inclui as drogas capazes de promover a adequada captação de iodo pela célula tireoideana, seja por estimular a transcrição adequada da proteína NIS ou por transportá-la do citoplasma para a membrana celular, onde ela é funcional.

\section{Ácido retinóico}

Os ácidos retinóicos são metabólitos ativos da vitamina A que regulam o crescimento e diferenciação de muitos tipos celulares, ligando-se a receptores nucleares específicos, os receptores do ácido retinóico (RAR) e os receptores do retinóide $\mathrm{X}$ ( $\mathrm{RXR}$ ), com três isoformas cada (alfa, beta e gama). Estudos recentes têm demonstrado que o ácido retinóico e análogos sintéticos são capazes de induzir uma rediferenciação em algumas linhagens de células de câncer de tireóide, sugerido pelo aumento da expressão da proteína NIS e incremento na captação celular de ${ }^{131} \mathrm{I}$. Além disto, a secreção de tireoglobulina $(\mathrm{Tg})$, desiodinases tireoideanas e fosfatase alcalina são estimuladas. O tipo tumoral (papilífero, folicular ou oxifílico) aparentemente não prediz a resposta terapêutica (13). Os estudos clínicos sugerem que 20 a $40 \%$ dos pacientes podem responder ao tratamento com 13 cis ácido retinóico (isotretinoina) (14-19). Foi demonstrado que a expressão do RAR-beta e o RXR-gama parece predizer uma resposta ao ligante retinóide natural (9cis $\mathrm{RA}$ ) e aos análogos RXR-seletivos sintéticos (LG346 ou TTNPB) em linhagens tumorais (13). Recentemente, estudou-se a possível correlação entre a expressão do RAR-beta no tumor e a presença de resposta favorável ao ácido retinóico (20). De cinco pacientes com carcinoma de tireóide pouco diferenciado submetidos ao ácido retinóico, a resposta clínica foi parcial em dois (aumento da captação de ${ }^{131} \mathrm{I}$ e redução da Tg sérica) e excelente em um (aumento da captação de ${ }^{131} \mathrm{I}$ associado com redução das metástases pulmonares). A imuno-histoquímica demonstrou uma redução significativa da expressão do 
RAR-beta nos tumores dos pacientes que não responderam ao AR e um aumento significativo no tumor que teve a melhor resposta (20).

\section{Análogos do ácido retinóico}

Sintéticos análogos do ácido retinóico, como o bexaroteno (LGD346 - Targretin), utilizado no tratamento do linfoma cutâneo de células $\mathrm{T}$, inibe a proliferação de células anaplásicas (ARO) que expressam intensamente RXR-gama. É provável que este efeito do Bexaroteno ocorra através da estimulação do Fator Inibidor de Leucemia (FIL), que inibe a proliferação das células ARO. Camundongos transplantados com células ARO desenvolvem tumores anaplásicos agressivos, cujo crescimento é inibido pelo bexaroteno (21).

\section{Inibidores da Histona Deacetilase (HDACI, Histone Deacetylase Inhibitors)}

As histonas são pequenas estruturas protéicas, de carga positiva, que compõem a maior parte da estrutura protéica do cromossoma, ligando-se fortemente ao DNA, de carga negativa, formando um complexo proteínaDNA condensado, desacetilado, na qual não ocorre transcrição. $\mathrm{O}$ relaxamento desta estrutura densa da histona pela acetil transferase é necessário para permitir a transcrição do DNA para RNA. O uso de HDACI, que inibe a desacetilação, leva a uma diferenciação celular. A incubação in vitro de células neoplásicas foliculares e anaplásicas de tireóide com depsipeptide (FR901228), um HDACI, aumentou a expressão da Tg e da NIS, com maior acúmulo de iodo intracelular (22).

\section{Agentes demetiladores}

A hipermetilação do DNA, através da enzima metil transferase, que ocorre freqüentemente na região promotora de genes, resulta numa ligação alterada de cofatores, podendo levar a uma diminuição da expressão gênica. Bloqueadores da metil transferase podem induzir reexpressão de genes supressores tumorais e de outros genes importantes para a facilitação do tratamento. 5-Azacitidina e 5-aza-2-deoxicitidinas, que são incorporadas ao DNA, inibem a metilação. Em linhagem de células de câncer de tireóide, o tratamento com 5-azacitidina foi capaz de restaurar a expressão da proteína NIS, bem como de outros importantes fatores de transcrição (23). Outras drogas que mostraram ser capazes de rediferenciar a célula que perdeu a capacidade de captar o iodo, incluem a lovastatina, o ácido valpróico e inibidores de enzima COX-2 $(5,24,25)$.

\section{QUIMIOTERAPIA COMBINADA COM ELEVAÇĀO DO TSH}

Levando em conta o aspecto de que os agentes citotóxicos são mais ativos contra células em mitose e que o TSH é o principal estimulador da proliferação das células foliculares tireoideanas, Pacini e cols. (11) utilizaram epirubicina e carboplatinum durante o estímulo com TSH, através da suspensão do TSH ou pela administração do TSH Recombinante, e observaram uma melhor eficácia da quimioterapia nestas condições. De 14 pacientes com carcinoma papilífero pouco diferenciado, um paciente teve recuperação completa, 5 tiveram uma recuperação parcial, 7 mantiveram a doença estável e 1 evolui para óbito (11). Tal abordagem parece ser promissora no tratamento das lesões pouco diferenciadas.

\section{DROGAS QUE AGEM EM ALVOS ESPECÍFICOS NO CÂNCER DE TIREÓIDE}

\section{RET quinase como alvo de drogas}

As proteínas tirosina quinases são enzimas que catalisam a transferência de fosfato do ATP para resíduos tirosina em polipeptídeos. Eles regulam a proliferação, sobrevida, diferenciação, função e motilidade celular. O gene RET quinase freqüentemente está mutado no carcinoma papilífero ou carcinoma medular de tireóide, levando a uma ativação de proliferação anormal. Foram desenvolvidos alguns compostos que têm a proteína RET como alvo (26) e são capazes de inibir a atividade quinase in vitro e in vivo. Entre estes, duas pirazolo-pirimidinas, PPl e PP2, e uma anilinoquinazolina, ZD6474, podem prevenir a transformação mediada pelo RET. São capazes de inibir a autofosforilação do RET/PTC e do RET mutados em MEN 2, bloqueando oncoproteínas, revertendo a morfologia transformada, proliferação autônoma e crescimento celular (26). O ZD6474 também é um inibidor do Receptor do Fator de Crescimento Epidérmico (EGFR) e do Receptor do Fator de Crescimento Vascular (VEGFR), em estudos clínicos avançados em função de sua atividade antiangiogênica. O ZD6474 tem efeito citostático em células de carcinoma de tireóide que possuem alelos RET oncogênicos (26).

\section{Proteína RAS como alvo de drogas}

O fenilacetato é um ácido graxo aromático natural derivado do metabolismo da fenilalanina, que inibe o crescimento celular por afetar o processamento postranslacional do RAS (figura 1). Em linhagem de células de câncer de tireóide humanos (folicular, papilar e células de Hürthle), o fenilacetato diminui o crescimento induzido e não induzido pelo TSH e induz o 


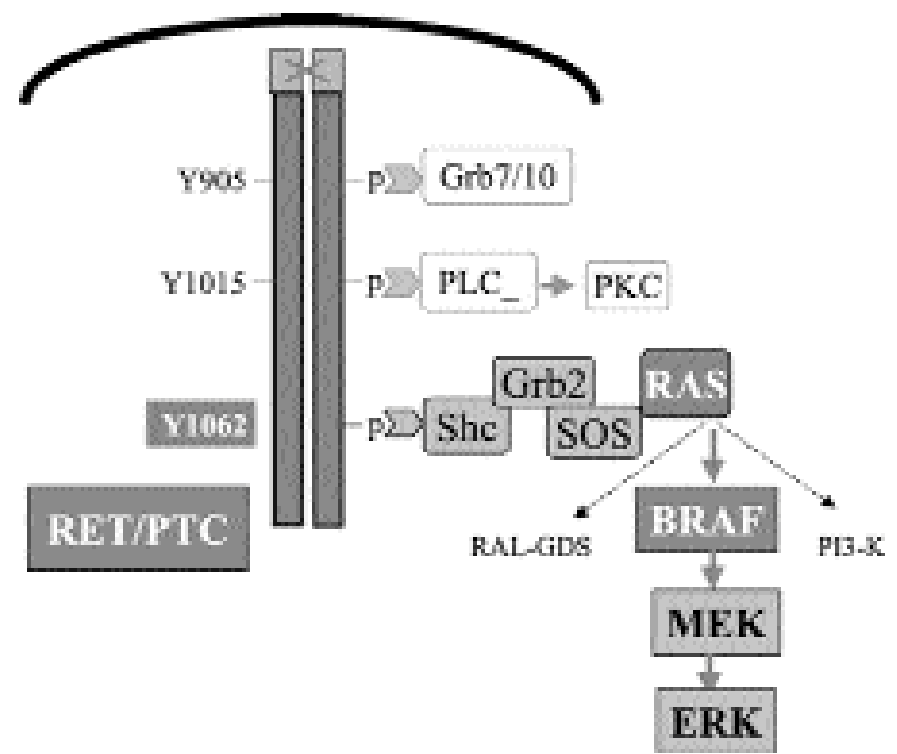

Figura 1. Sinalização molecular na proliferação da célula folicular tireoideana.

Adaptado de Fagin (30).

aumento da captação de ${ }^{131} \mathrm{I}$ e secreção de $\mathrm{Tg}$ (5). O fator de crescimento vascular endotelial foi inibido em todas as linhagens celulares, e o fenilacetato pode agir em cooperação com o ácido retinóico (27). A inibição da enzima farnesil transferase inibe a acumulação do Ras na membrana, reduzindo, assim, a sinalização celular. A manumicina, inibidor da farnesil transferase, reduz o número de células vivas em linhagem celular de carcinoma anaplásico, isoladamente ou em combinação com paclitaxel, doxorrubicina ou cisplatina $(28,29)$.

\section{Proteína B-RAF como alvo de drogas}

Existem três isoformas da serina-treonina quinase RAF: A-RAF, B-RAF e C-RAF. Todas ativam a fosforilação do MEK, mas B-RAF é a isoforma mais predominante na célula folicular tireoideana (30) (figura 1). A mutação $B R A F^{T 1796 A}$ é a mutação genética mais comum no CPT e representa cerca de 36 a $60 \%$ dos casos (31-38). A mutação $B R A F^{T 1796 A}$ é encontrada apenas no CPT e não existe superposição entre as mutações RET/PTC, BRAF e RAS, que juntas compõem $70 \%$ dos casos (31). A falta de concordância destas mutações fornece evidências genéticas fortes para a participação deste sistema na sinalização para transformação para CPT (figura 1).

Desta forma, a proteína BRAF representa um excelente alvo para o tratamento de câncer papilífero de tireóide em função do seu possível papel na iniciação da tumorigênese, pela sua alta prevalência e pela sua associação a tumores com estágio mais avançado, como carcinomas pouco diferenciados ou anaplásico
(30). O composto Bay 43-9006 é um potente e efetivo inibidor do RAF in vitro e em camundongos, e está sendo utilizado em ensaios clínicos para outras formas de câncer. O composto Bay 43-9006 apresenta um excelente perfil de segurança em seres humanos $(40,41)$. Trabalhos preliminares recentes mostram que o Bay 43-9006 pode inibir o crescimento e proliferação e induzir apoptose em células KAT-5, uma linhagem de células derivadas do CPT contendo uma mutação BRAF (42). Outros inibidores da RAF, na forma de pequenas moléculas inibidoras de quinases, bem como oligonucleotídeos antisense (Antisense ISIS 5132), estão em desenvolvimento (39).

\section{MEK como alvo de drogas}

Como observado na figura 1 , a proteína MEK está envolvida em várias vias oncogênicas (5), existindo duas isoformas, MEKl e MEK2, e a fosforilação de ambas pode ser inibida pelo composto CI01040. Esta droga, também conhecida como PD-184352, é a primeira droga que tem a MEK como alvo, candidata a ensaios clínicos (43). Um estudo recente demonstrou que o tratamento com inibidores de MEK pode restaurar a expressão da Tg e da proteína NIS (44).

\section{Angiogenese como alvo de drogas}

\section{Talidomida}

Além de ser considerada uma droga sedativa, a talidomida possui efeitos antitumorais, cujos mecanismos não são bem conhecidos, embora sua ação antiangiogênica

Arq Bras Endocrinol Metab vol $49 n^{\circ} 5$ Outubro 2005 
seja conhecida há mais de 10 anos (45). Baseados na importância da angiogênese no crescimento de tumores sólidos, sugeriu-se que o bloqueio angiogênico poderia reduzir o crescimento de carcinomas não diferenciados (46). Dados preliminares indicam que a talidomida tem potencial na redução da progressão da doença (46). O uso da talidomida se acompanha de um aumento da expressão do VEGF e da p53. No momento, existem estudos clínicos em fase II em carcinoma medular, folicular e papilíferos (5).

\section{Vias de apoptose (morte celular programa- da) como alvo de drogas}

As células do câncer de tireóide demonstram sensibilidade reduzida a morte celular, o que leva à capacidade das células em manter as alterações genéticas, mas continuar o crescimento (5). A apoptose é um processo organizado que leva à morte celular através de vias de sinalização específica (5). Existem drogas em desenvolvimento desenhadas para iniciar a apoptose, como o TRAIL (TNF-related apoptosis-inducing ligand). O TRAIL induz a apoptose em muitas células cancerosas humanas, embora elas possam ser resistentes a esta indução (47). A combinação do TRAIL com troglitazona, paclixatel e ciclo-heximida induziu apoptose em células de câncer tireoideano em concentrações subótimas não obtidas com o TRAIL isoladamente (47). A fosforilação da proteína $\mathrm{Bcl}-2$, um marcador molecular de várias neoplasias, está envolvido com resistência a apoptose, e a hiperexpressão do $\mathrm{Bcl}-2$ leva a proliferação de células tireoideanas cancerosas. $\mathrm{O}$ composto G3139, que resultou em morte celular e na redução da atividade e expressão da $\mathrm{Bcl}-2$ (48), poderá vir a ser útil no câncer de tireóide.

\section{Outras drogas potenciais para o tratamento do câncer de tireóide}

\section{Troglitazonas}

A troglitazona é um potente agonista do PPAR-gama (proliferator-activated receptor-gamma), que é um fator de transcrição que regula a diferenciação e crescimento celular. O PPAR-gama pode ter um papel na carcinogênese tireoideana uma vez que a translocação cromossômica PAX8-PPAR-gama é freqüentemente encontrada no carcinoma folicular de tireóide. O efeito antiproliferativo e de rediferenciação da troglitazona foi estudado em 6 linhagens de células tireoideanas cancerosas: TPC-1, FTC-133, FTC-236, FTC-238, XTC-1 (células de Hürthle) e ARO81-1 (células anaplásicas) (49). O PPAR-gama se expressou de forma variável nestes tecidos. A troglitazona inibiu de forma significativa o crescimento celular por parada do ciclo celular e apoptose, além de aumentar os níveis do RNAm da NIS em TPCl e FTC-133 (49). A droga RS-5444, que ativa a transcrição do PPAR-gama de forma mais potente que a tiazolidinediona, inibe a proliferação de células indiferenciadas de tireóide, bem como a formação de colônias. Além disto, o RS544 e o Taxol demonstram atividade sinergística anti-proliferativa em cultura de células (50). Desta forma, agonistas do PPAR-gama podem vir a ser agentes terapêuticos efetivos no tratamento de pacientes com câncer de tireóide que não respondem aos tratamentos convencionais. Assim como o PPAR-gama, o receptor do retinóide $\mathrm{X}$ se expressa de forma variável em linhagens de carcinomas de tireóide. A expressão destes receptores pode predizer a resposta das células de câncer de tireóide ao tratamento associado de retinóides e tiazolidinedionas. A expressão simultânea de RXR-gama e PPAR-gama, que agem como heterodímeros na transcrição celular, é necessária para a inibição máxima de crescimento e aumento de apoptose pelos ligantes. A combinação de pioglitazona e LG346 (um ligante específico do RXR) inibiu o crescimento celular em linhagens tumorais que expressavam o PPARgama e RXR-gama (51).

\section{Lovastatina}

Os inibidores da 3-Hydroxy-3-methylglutaryl coenzyme $A$ (HMG-CoA) redutase são capazes de promover a apoptose celular e a diferenciação em muitas células cancerosas, ações independentes da redução lipídica. Células tireoideanas indiferenciadas tratadas com lovastatina em doses elevadas foram levadas a apoptose, além de aumentar a secreção de Tg pelas células cancerosas (52). Estes resultados sugerem que outros inibidores da HMG-CoA redutase devem ser investigados como terapia diferenciadora.

\section{Inibidores da Cyclo-oxygenase-2 (COX-2)}

$\mathrm{O}$ ácido araquidônico e seus derivados prostaglandinas (PG) e tromboxane influenciam o crescimento de vários tumores. Análise por RT-PCR mostrou um aumento significativo de TXA(2) sintase em CPT comparado a tecido normal. A expressão da proteína COX-2 também estava aumentada no CPT (53). Estudos in vitro com o inibidor da COX-2, NS-398, mostrou inibição do crescimento tumoral, além do aumento nos níveis de COX-2 e da expressão do RNAm do fator-A de crescimento vascular endotelial. Estes resultados indicam que enzimas específicas da síntese de PG estão aumentadas no CPT, e que 
inibidores da COX-2 podem promover a apoptose e diminuir a angiogênese (53). Diversos ensaios, inclusive para pacientes com câncer de tireóide, estão sendo conduzidos com celecoxib, um inibidor da COX-2, isoladamente ou em combinação com outros agentes terapêuticos $(5)$.

\section{CONCLUSĀO}

A maioria (80 a $85 \%)$ das neoplasias de tireóide tem um ótimo prognóstico após o tratamento com tireoidectomia total e complementação com ${ }^{131}$ I. O grande desafio é a descoberta de novas medidas terapêuticas para aqueles pacientes com neoplasia mais agressiva, que perderam a diferenciação e para os quais até o momento não dispomos de um tratamento adequado. Com o melhor conhecimento da alteração de sinalização celular que leva a proliferação e crescimento tumoral, e o desenvolvimento de novas drogas dirigidas para atuar em determinado nível de sinalização, o quadro está se modificando, com perspectivas de aumento da sobrevida destes pacientes. Neste artigo, procuramos demonstrar as áreas mais excitantes na pesquisa do câncer de tireóide pouco diferenciado, com drogas inteligentes e mecanismo de ação cada vez melhor conhecidos.

\section{REFERÊNCIAS}

1. Pacini F, Cetani F, Miccoli P, Mancusi F, Ceccarelli $C$, Lippi $F$, et al. Outcome of 309 patients with metastatic differentiated thyroid carcinoma treated with radioiodine. World J Surg 1994; 18:600-4.

2. Schlumberger $M$, Challeton $C$, De Vathaire F, Travagli JP, Gardet P, Lumbroso JD, et al. Radioactive iodine treatment and external radiotherapy for lung and bone metastases from thyroid carcinoma. J Nucl Med 1996;37:598-605.

3. Schlumberger MJ. Diagnostic follow-up of well-differentiated thyroid carcinoma: historical perspective and current status. J Endocrinol Invest 1999;22(suppl. 11):37.

4. Casara D, Rubello D, Saladini G, Masarotto G, Favero A, Girelli ME, et al. Different features of pulmonary metastases in differentiated thyroid cancer: natural history and multivariate statistical analysis of prognostic variables. J Nucl Med 1993;34:1626-31.

5. Braga-Basaria $M$, Ringel MD. Clinical review 158: Beyond radioiodine - a review of potential new therapeutic approaches for thyroid cancer. J Clin Endocrinol Metab 2003;88:1947-60.

6. Shimaoka K, Schoenfeld DA, De Wys WD, Creech RH, De Conti R. A randomized trial of doxorubicin versus doxorubicin plus cisplatin in patients with advanced thyroid carcinoma. Cancer 1985;56:2155-60.

7. De Besi P, Busnardo B, Toso S, Girelli ME, Nacamulli D, Simioni $\mathrm{N}$, et al. Combined chemotherapy with bleomycin, adriamycin, and platinum in advanced thyroid cancer. J Endocrinol Invest 1991;14:475-80.

8. Ahuja S, Ernst H. Chemotherapy of thyroid carcinoma. J Endocrinol Invest 1987; 10:303-10.

9. Hoskin PJ, Harmer C. Chemotherapy for thyroid cancer. Radiother Oncol 1987;10:187-94.

10. Pacini F, Pinchera A, Mancusi F, Pollina L, Fontanelli G, Bevilacqua $G$, et al. Anaplastic thyroid carcinoma: a retrospective clinical and immunohistochemical study. Oncol Rep 1994;1:921-5.

11. Santini F, Bottici V, Elisei R, Montanelli L, Mazzeo S, Basolo $\mathrm{F}$, et al. Cytotoxic effects of carboplatinum and epirubicin in the setting of an elevated serum thyrotropin for advanced poorly differentiated thyroid cancer. J Clin Endocrinol Metab 2002;87:4160-5.

12. Leaf AN, Wolf BC, Kirkwood JM, Haselow RE. Phase II study of etoposide (VP-16) in patients with thyroid cancer with no prior chemotherapy: An Eastern Cooperative Oncology Group Study (E1385). Med Oncol 2000;17:47-51.

13. Haugen BR, Larson LL, Pugazhenthi U, Hays WR, Klopper JP, Kramer CA, et al. Retinoic acid and retinoid X receptors are differentially expressed in thyroid cancer and thyroid carcinoma cell lines and predict response to treatment with retinoids. J Clin Endocrinol Metab 2004;89:272-80.

14. Simon D, Koehrle J, Reiners C, Boerner AR, Schmutzler C, Mainz K, et al. Redifferentiation therapy with retinoids: therapeutic option for advanced follicular and papillary thyroid carcinoma. World J Surg 1998;22:569-75.

15. Grunwald F, Menzel C, Bender H, Palmedo H, Otte R, Fimmers R, et al. Redifferentiation therapy-induced radioiodine uptake in thyroid cancer. J Nucl Med 1998;39:1903-6.

16. Grunwald F, Pakos E, Bender H, Menzel C, Otte R, Palmedo $\mathrm{H}$, et al. Redifferentiation therapy with retinoic acid in follicular thyroid cancer. J Nucl Med 1998;39:1555-8.

17. Schmutzler C, Kohrle J. Retinoic acid redifferentiation therapy for thyroid cancer. Thyroid 2000;10:393-406.

18. Boerner AR, Petrich T, Weckesser E, Fricke H, Hofmann M, Otto $D$, et al. Monitoring isotretinoin therapy in thyroid cancer using 18F-FDG PET. Eur J Nucl Med Mol Imaging 2002;29:231-6.

19. Simon D, Korber C, Krausch M, Segering J, Groth P, Gorges R, et al. Clinical impact of retinoids in redifferentiation therapy of advanced thyroid cancer: final results of a pilot study. Eur J Nucl Med Mol Imaging 2002:29:775-82.

20. Coelho SM, Corbo R, Buescu A, Carvalho DP, Vaisman M. Ácido retinóico: uma terapia promissora para carcinoma diferenciado de tireoidiano desdiferenciado? Arq Bras de Endocrinol Metab 2003;47(2):190-7.

21. Klopper PJ, Haugen B. Synthetic Retinoids as a Treatment for Anaplastic Carcinoma. Endocrine Society Meeting, San Diego, 2005. 
22. Kitazono M, Robey R, Zhan Z, Sarlis NJ, Skarulis MC, Aikou $\mathrm{T}$, et al. Low concentrations of the histone deacetylase inhibitor, depsipeptide (FR901228), increase expression of the $\mathrm{Na}^{+} / \mathrm{I}^{-}$symporter and iodine accumulation in poorly differentiated thyroid carcinoma cells. J Clin Endocrinol Metab $2001 ; 86: 3430-5$

23. Venkataraman $G M$, Yatin $M$, Marcinek $R$, Ain KB. Restoration of iodide uptake in dedifferentiated thyroid carcinoma: Relationship to human $\mathrm{Na}^{+} / \mathrm{I}^{-}$symporter gene methylation status. J Clin Endocrinol Metab 1999;84:2449-57.

24. Fortunati N, Catalano MG, Arena K, Brignardello $E$, Piovesan A, Boccuzzi $G$. Valproic acid induces the expression of the $\mathrm{Na}^{+} / \mathrm{I}^{-}$symporter and iodine uptake in poorly differentiated thyroid cancer cells. J Clin Endocrinol Metab 2004;89(2):1006-9.

25. Wang CY, Zhong WB, Chang TC, Lai SM, Tsai YF. Lovastatin, a 3-hydroxy-3-methylglutaryl coenzyme A reductase inhibitor, induces apoptosis and differentiation in human anaplastic thyroid carcinoma cells. J Clin Endocrinol Metab 2003;88(7):3021-6.

26. Carlomagno F, Santoro M. Identification of RET kinase inhibitors as potential new treatment for sporadic and inherited thyroid cancer. J Chemother 2004; 16(suppl. 4):49-51.

27. Eigelberger MS, Wong MG, Duh QY, Clark OH. Phenylacetate enhances the antiproliferative effect of retinoic acid in follicular thyroid cancer. Surgery 2001;130:9315.

28. Yeung SC, Xu G, Pan J, Christgen M, Bamiagis A. Manumycin enhances the cytotoxic effect of paclitaxel on anaplastic thyroid carcinoma cells. Cancer Res 2000;60:650-6

29. Xu G, Pan J, Martin C, Yeung SC. Angiogenesis inhibition in the in vivo antineoplastic effect of manumycin and paclitaxel against anaplastic thyroid carcinoma. $\mathbf{J}$ Clin Endocrinol Metab 2001;86:1769-77.

30. Fagin JA. How thyroid tumors start and why it matters: Kinase mutants as targets for solid cancer pharmacotherapy. J Endocrinol 2004; 183:249-56.

31. Kimura ET, Nikiforova MN, Zhu Z, Knauf JA, Nikiforov YE, Fagin JA. High prevalence of BRAF mutations in thyroid cancer: genetic evidence for constitutive activation of the RET/PTC-RAS-BRAF signaling pathway in papillary thyroid carcinoma. Cancer Res 2003;63:1454-7.

32. Trovisco V, Vieira de Castro I, Soares P, Maximo V, Silva $P$, Magalhães J, et al. BRAF mutations are associated with some histological types of papillary thyroid carcinoma. J Pathol 2004;202:247-51.

33 Nikiforova MN, Kimura ET, Gandhi M, Biddinger PW, Knauf JA, Basolo $F$, et al. BRAF mutations in thyroid tumors are restricted to papillary carcinomas and anaplastic or poorly differentiated carcinomas arising from papillary carcinomas. J Clin Endocrinol Metab 2003:88:5399-404.

34. Fukushima T, Suzuki S, Mashiko M, Ohtake T, Endo Y, Takebayashi $Y$, et al. BRAF mutations in papillary carcinomas of the thyroid. Oncogene 2003;22:6455-7.

35. Namba H, Nakashima M, Hayashi T, Hayashida N, Maeda S, Rogounovitch TI, et al. Clinical implication of hot spot BRAF mutation, V599E, in papillary thyroid can- cers. J Clin Endocrinol Metab 2003;88:4393-7.

36. Xu X, Quiros RM, Gattuso P, Ain KB, Prinz RA. High prevalence of BRAF gene mutation in papillary thyroid carcinomas and thyroid tumor cell lines. Cancer Res 2003;63:4561-7.

37. Soares P, Trovisco V, Rocha AS, Lima J, Castro P, Preto A, et al. BRAF mutations and RET/PTC rearrangements are alternative events in the etiopathogenesis of PTC. Oncogene 2003:22:4578-80.

38. Cohen $\mathrm{Y}$, Xing M, Mambo E, Guo Z, Wu G, Trink B, et al. BRAF mutation in papillary thyroid carcinoma. J NatI Cancer Inst 2003;95:625-7.

39. Fagin JA. Challenging dogma in thyroid cancer molecular genetics - Role of RET/PTC and BRAF in tumor Initiation. J Clin Endocrinol Metab 2004;89:4264-6.

40. Bollag G, Freeman S, Lyons JF, Post LE. Raf pathway inhibitors in oncology. Curr Op Invest Drugs 2003;4:1436-41.

41. Lee JT, McCubrey JA. BAY-43-9006. Bayer/Onyx. Curr Op Invest Drugs 2003;4:757-63.

42. Kumar MS, Moore KE, Brose MS. Functional analysis of BRAF in a papillary thyroid cancer cell line. Thyroid 2004; $14: 712$

43. Sebolt-Leopold JS. MEK inhibitors: a therapeutic approach to targeting the Ras-MAP kinase pathway in tumors. Curr Pharmac Design 2004;10:1907-14.

44. Knauf JA, Kuroda H, Basu S, Fagin JA. RET/PTC-induced dedifferentiation of thyroid cells is mediated through Y1062 signaling through SHC-RAS-MAP kinase. Oncogene 2003;22:4406-12.

45. D'Amato RJ, Loughnan MS, Flynn E, Folkman J. Thalidomide is an inhibitor of angiogenesis. Proc Natl Acad Sci USA 1994:91:4082-5.

46. Bauer AJ, Terrell R, Doniparthi NK, Patel A, Tuttle RM, Saji $M$, et al. Vascular endothelial growth factor monoclonal antibody inhibits growth of anaplastic thyroid cancer xenografts in nude mice. Thyroid 2002;12(11):953-61.

47. Park JW, Wong MG, Lobo M, Hyun WC, Duh QY, Clark OH. Modulation of tumor necrosis factor-related apoptosisinducing ligand-induced apoptosis by chemotherapy in thyroid cancer cell lines. Thyroid 2003;13(12):1103-10.

48. Morris MJ, Tong WP, Cordon-Cardo C, Drobnjak M, Kelly WK, Slovin SF, et al. Phase I trial of BCL-2 antisense oligonucleotide (G3139) administered by continuous intravenous infusion in patients with advanced cancer. Clin Cancer Res 2002;8:679-83.

49. Park JW, Zarnegar R, Kanauchi H, Wong MG, Hyun WC, Ginzinger DG, et al. Troglitazone, the peroxisome proliferator-activated receptor-gamma agonist, induces antiproliferation and redifferentiation in human thyroid cancer cell lines. Thyroid 2005; 15(3):222-31.

50. Grebe SF. Thiazolidinediones, PPAR gamma and thyroid cancer. Frontiers in Thyroid Cancer 2005, ATA, April 2005, Baltimore, USA.

51. Klopper JP, Hays WR, Sharma V, Baumbusch MA, Hershman JM, Haugen BR. Retinoid $X$ receptor- $\gamma$ and peroxi- 
some proliferator-activated receptor- $\gamma$ expression predicts thyroid carcinoma cell response to retinoid and thiazolidinedione treatment. Mol Cancer Ther 2004;3:101 1-20.

52. Wang CY, Zhong WB, Chang TC, Lai SM, Tsai YF. Lovastatin, a 3-Hydroxy-3-methylglutaryl Coenzyme A reductase inhibitor, induces apoptosis and differentiation in human anaplastic thyroid carcinoma cells. J Clin Endocrinol Metab 2003;88/7:3021-6.
53. Kajita S, Ruebel KH, Casey MB, Nakamura N, Lloyd RV. Role of COX-2, thromboxane A2 synthase, and prostaglandin 12 synthase in papillary thyroid carcinoma growth. Mod Pathol 2005;18(2):221.

Endereço para correspondência:

Hans Graf

Rua Solimōes 1184

80810-070 Curitiba, PR

E-mail: hansgraf@bsi.com.br 